\title{
EL OESTE CHAQUEÑO. UNA APROXIMACIÓN A LA RELACIÓN HOMBRE- NATURALEZA.
}

Viviana Claudia Pertile

vpertile@hum.unne.edu.ar

Instituto de Geografía- UNNE

Alejandra H. Torre Geraldi

ageraldi@bib.unne.edu.ar

Instituto de Geografía- UNNE- IIGHI- Conicet

\section{Resumen}

Desde la última década del Siglo XX el espacio agrario argentino se ha caracterizado por manifestar profundos procesos de cambio y significativa reestructuración propios del modelo capitalista, logrando la apertura del mercado graneario hacia el resto del mundo.

Estas transformaciones han impactado no sólo en los tradicionales espacios productivos, sino también en áreas periféricas con inferior crecimiento. La provincia del Chaco, una de las jurisdicciones extra pampeanas que incorporó y "absorbió" estos cambios, presenta en la actualidad un proceso de crisis interna agraria como resultado de los nuevos manejos existentes entre el hombre y el medio; incremento de la pobreza, exclusión y expulsión social, así como el deterioro del ambiente, a partir de la implementación de nuevas prácticas y deseos de expansión territorial que han desfigurado el tradicional espacio agrario chaqueño. Es por ello que, ante lo expuesto, el presente trabajo pretende abordar las particularidades que adquiere la relación hombre- naturaleza en el sector occidental del Chaco a fines del siglo XX.

Palabras Clave: productores- deterioro ambiental- prácticas productivas- nuevas tecnologías.

\section{The West Chaco. An approximation to the relation Man - nature}

\begin{abstract}
Since the last decade of the twentieth century, Argentina's agricultural area has been characterized by expressing profound processes of change and significant restructuring of the capitalist model distinctive, making the opening of the grain market to the rest of the world.

These changes have impacted not only the traditional production spaces, but also in marginal areas with less growth. The province of Chaco, a jurisdiction outside the Pampas you added, and "absorbed" these changes, has now an internal process of agrarian crisis as a result of new procedures involving humans and the environment, with increased poverty, social exclusion and expulsion, also the deterioration of the environment, from the implementation of new practices and desire for territorial expansion that has transformed the traditional Chaco agricultural area. That is why, given the above, this paper aims to study the peculiarities of the relationship between man and nature in the western Chaco late twentieth century.
\end{abstract}

Keywords: producers, environmental degradation, production practices and new technologies

Publicado en formato digital: Mgter. Viviana Claudia Pertile y Prof. Alejandra H. Torre Geraldi. EL OESTE CHAQUEÑO. UNA APROXIMACIÓN A LA RELACIÓN HOMBRE-NATURALEZA. Revista Geográfica Digital. IGUNNE. Facultad de Humanidades. UNNE. Año 8. No 16. Julio - Diciembre 2011. ISSN 1668-5180 Resistencia, Chaco. En: http://hum.unne.edu.ar/revistas/geoweb/default.htm 
Revista Geográfica Digital. IGUNNE. Facultad de Humanidades. UNNE. Año 8. № 16. Julio - Diciembre 2011.

\section{Introducción}

El agro argentino, desde fines de la década del ' 80 , se encuentra atravesando procesos de significativa reestructuración que incluyen cambios de mercados como resultado de la apertura a la economía mundial, procesos de modernización tecnológica, modificaciones en la función del Estado en términos de redistribución de los excedentes agrarios. Esta nueva situación ha determinado el aumento de requerimientos de capital para poder continuar en la producción Bidaseca, Gras y Mariotti (2000). Por otra parte, las transformaciones naturales, económicas, y sociales que caracterizaron a la década del '80 tuvieron un notable impacto en el ambiente y en las condiciones de vida de la población, a tal punto que crecientes grupos sociales se vieron excluidos de las posibilidades de atender sus necesidades básicas Carlevari (1996). Dichas transformaciones también abarcaron a las poblaciones dedicadas al agro y la evidencia más notable fue el deterioro del nivel de vida del pequeño agricultor, como consecuencia de la pérdida de productividad, a lo que debemos sumarle la disminución del empleo de la mano de obra contratada por los grandes productores - dada la alta tecnificación- lo cual determina una disminución en los ingresos de las familias dedicadas a la agricultura, sobre parcelas medianas o pequeñas. Estos cambios han sido acompañados por un proceso de concentración de la propiedad agropecuaria y una sobreexplotación de los recursos naturales. Pertile (2003).

En otras palabras, éstos hechos determinaron la intensificación de los procesos productivos primarios y la expansión de la agricultura hacia nuevas áreas, dada las características edafoclimáticas de la provincia del Chaco -los recursos edáficos son variados especialmente si consideramos su composición y potencialidades y las características climáticas-. Mas del $90 \%$ de la superficie provincial se encuentra cubierta por suelos denominados climatogénicos Bonfils (1970) [1] predominando en el sector Sudoeste los suelos castaños rojizos o pardos rojizos, cuyas características principales provienen principalmente de la influencia de la vegetación xerófila y del clima con precipitaciones que oscilan entre 300 y 600 milímetros anuales, se desarrollan en el noroeste chaqueño.

Es por ello que, ante lo expuesto, nuestra intención es abordar las particularidades que adquiere la relación "Hombre - Recursos Naturales" en el sector Oeste de la provincia del Chaco, en las dos últimas décadas.

Publicado en formato digital: Mgter. Viviana Claudia Pertile y Prof. Alejandra H. Torre Geraldi. EL OESTE CHAQUEÑO. UNA APROXIMACIÓN A LA RELACIÓN HOMBRE-NATURALEZA. Revista Geográfica Digital. IGUNNE. Facultad de Humanidades. UNNE. Año 8. № 16. Julio - Diciembre 2011. ISSN 1668-5180 Resistencia, Chaco. En: http://hum.unne.edu.ar/revistas/geoweb/default.htm 
Revista Geográfica Digital. IGUNNE. Facultad de Humanidades. UNNE. Año 8. № 16. Julio - Diciembre 2011.

ISSN 1668-5180 Resistencia, Chaco

\section{La Sociedad y su relación con el Espacio Geográfico Chaqueño}

El espacio geográfico, objeto de estudio, es un área propicia para el desarrollo de actividades agropecuarias, por cuanto presenta condiciones edafoclimáticas, no óptimas pero al menos adecuadas para dichas actividades.

Si este medio natural no ofrece gran resistencia a las prácticas agropecuarias, es entonces, la relación Sociedad - Naturaleza la que nos va a caracterizar lo que para Carl Sauer (1941) sería el "Paisaje natural", es decir la versión geográfica de la economía del grupo a partir de las cuales se establecen las distintas áreas con sus respectivas actividades humanas, donde el contacto con el medio es directo y esencial. Este ambiente o hábitat es el producto de la conducta de un grupo dado sobre ese hábitat y ésta no responde a un condicionante físico sino a hábitos adquiridos [2] (Sauer, Op cit). En tal sentido Adrián Zarrilli (2000) coincide en algunos puntos con Sauer, al manifestar que existe una estrecha relación entre sociedad y medio ambiente, ya que los mismos son respectivamente subsistemas conformados del sistema global que se condicionan entre sí. Por lo tanto el desarrollo histórico de una sociedad depende en gran medida de su base ecológica y de sus recursos naturales, mientras que el tipo y grado de desarrollo afectan directamente esa base ambiental.

En este sentido, los productores agropecuarios continúan arraigados en sus hábitos y costumbres -producto de sus actitudes y destrezas adquiridas o heredadas- dedicándose al cultivo de la tierra.

Por su parte Harvey (2000) manifiesta que hay que "reconocer las múltiples cualidades objetivas que el tiempo y el espacio pueden expresar y el rol que las prácticas humanas desarrollan en su construcción (...) La objetividad del tiempo y el espacio está dada, en cada caso, por las prácticas materiales de la reproducción social y, si tenemos en cuenta que estas últimas varían geográfica e históricamente, sabremos que el tiempo social y el espacio social están construidos de manera diferencial". En definitiva cada modo de producción o formación social particular encarnará un conjunto de prácticas y conceptos del tiempo y del espacio.

Por otra parte, como lo expresa Reboratti (1999) cada cultura constituye una forma integral de vida, que plantea su propia y específica manera de resolver las relaciones esenciales (por ser ineludibles). Estas son: la de cada individuo consigo mismo; con los miembros de su comunidad, con las otras comunidades y con la naturaleza. Es decir que la 
forma de relación con el medio natural no es única y absoluta. Desde el momento en que la naturaleza- desde el medio ambiente hasta la naturaleza orgánica del hombre- es afectada por las relaciones sociales de producción, estos procesos biológicos son sobredeterminados por los procesos históricos en que el hombre o la naturaleza se insertan.

La peculiaridad física del medio ambiente, objeto de estudio, la historia de sus prácticas productivas y sociales, así como sus intercambios culturales en la historia, han condicionado la capacidad productiva, la división del trabajo, los niveles de consumo y la producción de excedentes comercializables. La intervención más o menos fuerte del capital y de los estados nacionales, modifican estas modalidades de transformación del medio ambiente y de los estilos culturales, por la introducción de nuevas técnicas y nuevos modelos productivos. Por lo tanto, el impacto y deterioro sobre el medio ambiente, producto de las prácticas desmedidas de los empresarios dedicados a las actividades agropecuarias [3], ha sido significativo y no puede ser comprendido en su complejidad ni resuelto con eficacia sin el concurso e integración de distintos campos del saber.

Las prácticas de uso de los recursos dependen del sistema de valores de las comunidades, de la significación cultural de sus recursos, de la lógica social y ecológica de sus prácticas productivas, también de su capacidad para asimilar a éstas conocimientos científicos y técnicos modernos. Así, el vínculo sociedad-naturaleza debe entenderse como una relación dinámica, la cual depende de la articulación histórica de los procesos tecnológicos y culturales que especifican las relaciones sociales de producción de una formación socioeconómica, así como la forma particular de desarrollo integrado o de degradación destructiva de sus fuerzas productivas (Reboratti, Op.cit).

En definitiva y retomando la idea de Sauer, respecto a que el medio ambiente es cambiante en tanto es un término de una apreciación cultural, el espacio rural objeto de estudio, no escapa a las transformaciones ocurridas en las últimos dos décadas. En este caso las referidas a cambios ocurridos en el agro argentino y sus vinculaciones con procesos sociales y económicos en el nivel mundial conocido como "globalización" [4], entendida según Garriaca, (1996: 112) "como la consolidación de una economía mundial que antecede a la acción de los actores o como una construcción social donde intervienen actores o sujetos sociales muy diferentes entre sí y con distintas capacidades de negociación". 


\section{La peculiaridad ambiental del territorio agrícola chaqueño: sus consecuencias a partir del corrimiento de la isohieta de 800 milímetros.}

Podríamos decir que el territorio agrícola chaqueño, cabría en las conceptualizaciones de Corboz (2001), al manifestar que es el resultado de diversos procesos (naturales y humanos) que interactúan a través del tiempo para dar como resultado "un producto" en tanto tienen una forma y personalidad que lo hacen único e irrepetible.

A partir de estudios realizados Pertile (2004) [5], se ha concluido que las tres últimas décadas fueron más húmedas de lo que se considera normal. La isohieta de $800 \mathrm{~mm}$ se desplazó unos $150 \mathrm{~km}$. hacia el oeste; este hecho natural posibilitó la agricultura en lugares en los que anteriormente no era posible, dada la escasez de precipitaciones. En otras palabras, estamos ante la presencia de la incorporación de nuevas tierras al circuito productivo en el Oeste de la provincia del Chaco, con el consiguiente incremento de la producción. Esta expansión del área agrícola se caracterizó por la sobreexplotación del monte nativo; la presencia de grandes productores, algunos de los cuales tienen entre mil y cinco mil hectáreas dedicadas a la agricultura; la multiplicación de las desmotadoras privadas en Chaco y el deterioro económico de las Cooperativas y Pymes; la tecnificación agrícola, representada fundamentalmente por la cosecha mecánica, gracias al abaratamiento de las maquinarias nacionales e importadas, entre otras características (Pertile, Op cit)

Los primeros en ocupar las nuevas tierras agrícolas fueron agricultores chaqueños ávidos de cultivar espacios más grandes. En un principio fue el cultivo del algodón, cuando el valor del textil era rentable, posteriormente las malas condiciones climáticas y la caída de los precios hicieron que este cultivo fuera reemplazado, por el girasol, la soja, el trigo, el sorgo y el maíz, permitiendo de este modo la diversificación de la agricultura.

Posteriormente, el auge de la soja y la posibilidad de sembrarla sobre el rastrojo del trigo atrajo a grandes productores de Santa Fe, Córdoba y hasta de Buenos Aires. Estos productores vieron la posibilidad de incrementar sus ganancias en poco tiempo; situación que determinó la ocupación de los campos que estaban bajo producción y también llevó a incorporar nuevas tierras; en consecuencia, es que se hizo un uso y abuso de las tierras ocupadas por la vegetación nativa conocida como monte chaqueño-santiagueño, sin importar la destrucción ecológica y la sobreexplotación de los recursos naturales. Las

Publicado en formato digital: Mgter. Viviana Claudia Pertile y Prof. Alejandra H. Torre Geraldi. EL OESTE CHAQUEÑO. UNA APROXIMACIÓN A LA RELACIÓN HOMBRE-NATURALEZA. Revista Geográfica Digital. IGUNNE. Facultad de Humanidades. UNNE. Año 8. No 16. Julio - Diciembre 2011. ISSN 1668-5180 Resistencia, Chaco. En: http://hum.unne.edu.ar/revistas/geoweb/default.htm 
Revista Geográfica Digital. IGUNNE. Facultad de Humanidades. UNNE. Año 8. № 16. Julio - Diciembre 2011. ISSN 1668-5180 Resistencia, Chaco

topadoras se convirtieron en herramientas comunes en el paisaje agrario y surgieron de ese modo las grandes extensiones de tierras en condiciones de ser usadas con fines agrícolas.

De este modo, se desarrollaron "patrones productivos [6] orientados a maximizar las ganancias de corto plazo, sin considerar las condiciones de conservación y regeneración de los recursos renovables, induciendo procesos que han degradado la calidad ambiental y los niveles de vida". Zarrilli, (2000).

\section{La situación ambiental del Oeste chaqueño y sus antecedentes en el uso del suelo.}

En los procesos de explotación de los recursos naturales se produce invariablemente algún tipo de efecto sobre el ambiente Reboratti (1999). En este sentido es que miramos la situación actual del ambiente chaqueño, al decir de Reboratti, analizando no lo que el ambiente ofrece a la sociedad sino los efectos que sobre él tiene la sociedad. Reafirmando la idea planteada, respecto de los "hábitos adquiridos" debemos decir que la situación actual a la que hacemos referencia, tiene sus antecedentes en los años 1880, con las primeras conquistas y colonización de nuestra región chaqueña. El aprovechamiento de la riqueza forestal se convirtió en el primer estímulo económico que gravitó en la penetración de la planicie chaqueña; pero esta explotación sólo fue con fines extractivos, sin intención de originar asentamientos poblacionales permanentes [7]. La característica fundamental de este tipo de aprovechamiento del medio natural fue el gran movimiento de mano de obra que realizaba dejando tras de si una vasta superficie deforestada de bosques xerófilos, cuya reposición natural es dificultosa Bruniard (1978). Posteriormente, y en los claros dejados por esta actividad, se desarrolló la actividad agrícola que perduraría hasta nuestros días. Esta se inicia a fines del siglo pasado, cuando en los años 1894-1895, el Gobierno Nacional distribuyó semillas de algodón importadas de los Estados Unidos a colonos del Chaco y Formosa, constituyéndose ese hecho en el hito inicial del cultivo de algodón en nuestra provincia, cultivo que en siembras experimentales tuvo buenos resultados Miranda, G: (1955), iniciándose en el año 1895 en el viejo Fortín San Carlos, cerca de la Escondida, distante 60 kilómetros al Noroeste de Resistencia. Este cultivo demostró, desde un principio, ambientarse muy bien a las condiciones agroecológicas predominantes en la zona. Desde entonces y prácticamente en forma ininterrumpida hasta la actualidad, la agricultura se ha constituido en uno de los pilares de la economía provincial chaqueña a tal punto que, como ya lo manifestáramos, hubo una intensificación de los procesos productivos primarios,

Publicado en formato digital: Mgter. Viviana Claudia Pertile y Prof. Alejandra H. Torre Geraldi. EL OESTE CHAQUEÑO. UNA APROXIMACIÓN A LA RELACIÓN HOMBRE-NATURALEZA. Revista Geográfica Digital. IGUNNE. Facultad de Humanidades. UNNE. Año 8. № 16. Julio - Diciembre 2011. ISSN 1668-5180 Resistencia, Chaco. En: http://hum.unne.edu.ar/revistas/geoweb/default.htm 
acompañados por la expansión de la agricultura hacia nuevas áreas, de extrema fragilidad ambiental.

\section{Situación Actual}

De modo que esta relación hombre-naturaleza, ha generado una serie de problemas [8], ampliamente conocidos tanto por los círculos académicos, como políticos, gubernamentales, no gubernamentales, religioso, entre otros; y sobre los cuales se debe trabajar en pos de lograr un adecuado manejo de los recursos naturales de nuestra provincia. Entre los problemas ambientales más destacados debemos mencionar: - la degradación del medio debido al manejo inapropiado del recurso "suelo". Práctica que se viene haciendo desde comienzos del desarrollo de las actividades primarias, ya sea por los sistemas agrícolas del monocultivo del algodón y actualmente de la soja, como por la sobrecarga de animales en terrenos poco aptos. Por otra parte, existe una deforestación incontrolada, en consecuencia nos encontramos con especies en extinción y por ende, con pérdida de la biodiversidad, a lo cual debemos agregar sequías e inundaciones agravadas por el desmonte mencionado; Otra de las cuestiones se relaciona con el Inadecuado uso de biocidas y fertilizantes los cuales pueden ocasionar graves perjuicios por contaminación de las aguas superficiales y subterráneas, del suelo y del aire.

En cuanto a los problemas económico-productivos, se ha detectado la falta de sistemas productivos primarios diversificados e integrados, que eviten la pérdida de productividad de los establecimientos agropecuarios; existe una presión fiscal y una legislación inadecuada, que afecta a la producción de los minifundistas y de pequeños productores; Es también notable la falta o escasez de asesoramiento técnico para productores pequeños y medianos, a los efectos de informar sobre el uso y manejo adecuado del suelo, para producir conservando. Por otra parte, el productor está invadido por campañas propagandísticas que hacen referencia a tecnologías de insumos; $y$, entre otros debemos mencionar como problema económico-productivo, la ubicación lejana a centros comerciales y puertos, con lo cual los fletes son bastante caros.

Finalmente, y como corolario de lo mencionado con anterioridad, debemos hacer referencia a los problemas sociales. Uno de los más notorios es - baja calidad de vida de los pequeños productores rurales, caracterizada por un alto índice de Necesidades Básicas Insatisfechas (educación - salud - seguridad.) hecho que determina la expulsión de productores rurales a las ciudades en busca de trabajo y mejores condiciones de vida, 
quienes terminan viviendo en condiciones de marginalidad; esto se traduce a su vez en otro problema, que tiene que ver con la falta de reconocimiento de su identidad cultural; otro problema es la ineficiente e irregular distribución de la tierra pública, lo cual genera una inestabilidad en las prácticas primarias y finalmente, mencionamos a la falta de mano de obra capacitada y/o reentrenada en oficios rurales, con lo cual podríamos sintetizar diciendo que estamos ante la presencia de un alto grado de pobreza que está padeciendo la población que se dedica a las actividades primarias en pequeñas escalas.

\section{Conclusiones}

Durante las dos últimas décadas el problema del deterioro ambiental ha estado en el centro de muchas discusiones académicas. Podría decirse que dicho deterioro está muy relacionado con la visión de la naturaleza como algo que tiene un valor instrumental -como a un medio a través del cual el ser humano puede alcanzar sus fines- en el marco de las relaciones dinámicas que se plantean entre los procesos históricos, tecnológicos y sociales; y con el no reconocimiento de su valor intrínseco.

Desde el punto de vista de las relaciones Hombre - Naturaleza en la provincia del Chaco en los últimos años podemos concluir lo siguiente:

- El uso inadecuado de los recursos naturales, ha desencadenado procesos de degradación de los mismos, en muchos casos graves, con efectos negativos en las condiciones de vida de las poblaciones, migraciones internas con concentraciones en núcleos urbanos y pérdidas de pautas culturales.

- La expansión de la frontera agropecuaria, bajo un modelo productivo de carácter neoliberal, con el consiguiente cambio de cobertura ha incluido la pérdida de hábitat, especies e información genética. Asimismo la conservación del suelo y el agua se han visto afectados, manifestándose en degradación física del suelo, aumento del escurrimiento, transporte de sedimentos, compactación, salinización, pérdidas de materias orgánicas y nutrientes.

- Los sistemas productivos tradicionales (algodonero, ganadería extensiva bovina y caprina, explotación de los bosques nativos, etc.), encuentran limitaciones para su

Publicado en formato digital: Mgter. Viviana Claudia Pertile y Prof. Alejandra H. Torre Geraldi. EL OESTE CHAQUEÑO. UNA APROXIMACIÓN A LA RELACIÓN HOMBRE-NATURALEZA. Revista Geográfica Digital. IGUNNE. Facultad de Humanidades. UNNE. Año 8. No 16. Julio - Diciembre 2011. ISSN 1668-5180 Resistencia, Chaco. En: http://hum.unne.edu.ar/revistas/geoweb/default.htm 
Revista Geográfica Digital. IGUNNE. Facultad de Humanidades. UNNE. Año 8. № 16. Julio - Diciembre 2011. ISSN 1668-5180 Resistencia, Chaco

desarrollo por baja productividad y rentabilidad condicionada por factores ambientales, estructurales, sanitarios, tecnológicos y de acceso a mercados.

- La introducción de nuevas tecnologías de producción, nuevos cultivos, y aportes de capital, han provocado un aumento en la actividad económica de la región que no se visualiza en un mejoramiento sostenido de los parámetros de equidad social.

- Las posibilidades que brindan los servicios ambientales no estan suficientemente internalizadas en la población y de manera incipiente en algunos sectores de los estados provinciales. En este aspecto dichas posibilidades son muy amplias, por la gran oferta y fragilidad que presentan algunos de los ecosistemas para las producciones tradicionales y a los cuales es necesario encontrar alternativas rentables que faciliten la aplicación de criterios de restricción o cambio de uso

- El análisis de los sistemas productivos generalmente se ha efectuado a partir de enfoques parciales (tecnológicos, económicos) de corto plazo, dejando de lado el accionar interdisciplinario que incluya la percepción ambiental y social en el contexto del necesario ordenamiento territorial de largo plazo.

Por último, es preciso destacar que la región cuenta con un alto potencial de recursos naturales, para la implementación de alternativas productivas que solo demandan la necesaria investigación de los sistemas productivos acordes a las condiciones ambientales particulares de la región, esto, sumado a decisiones políticas adecuadas y al compromiso de todos los actores involucrados, permitirían asegurar la sostenibilidad, competitividad, acceso a mercados y equidad social.

Finalmente y a modo de cierre nos cabe decir que, es preciso llamar a la reflexión acerca de las motivaciones que mueven a las sociedades a realizar un uso y abuso de la naturaleza para la satisfacción no sólo de sus necesidades, sino también de sus aspiraciones y deseos; por lo tanto, es aquí donde se debe plantear la idea de sustentabilidad, no solo de lo natural sino también de lo humano.

Publicado en formato digital: Mgter. Viviana Claudia Pertile y Prof. Alejandra H. Torre Geraldi. EL OESTE CHAQUEÑO. UNA APROXIMACIÓN A LA RELACIÓN HOMBRE-NATURALEZA. Revista Geográfica Digital. IGUNNE. Facultad de Humanidades. UNNE. Año 8. No 16. Julio - Diciembre 2011. ISSN 1668-5180 Resistencia, Chaco. En: http://hum.unne.edu.ar/revistas/geoweb/default.htm 
Revista Geográfica Digital. IGUNNE. Facultad de Humanidades. UNNE. Año 8. № 16. Julio - Diciembre 2011.

\section{Notas}

[1] Suelos climatogénicos: son aquellos "cuya evolución emana primordialmente de la influencia del clima y de los agentes bióticos..."Bonfils, Constante G. "Los Recursos Edáficos". En Boletín de la Sociedad Argentina de Botánica, Vol. XI, Buenos Aires, 1970.

[2]Para Sauer, el medio ambiente es un término de una apreciación cultural, es un valor en la historia de la cultura; en tanto el hábito es un aprendizaje activo común a un grupo, pero también puede ser inventado, adquirido o impuesto.

[3]Respondían a un modelo productivo agroexportador/neocapitalista, caracterizado por las innovaciones tecnológicas, los juegos financieros y de mercado y cuyo esquema de producción se caracteriza por el alto aporte de capital y tecnología en la que confluyen procesos naturales y sociales de diferentes órdenes.

[4] Esta globalización se torna más visible a partir del año 1989 con la desintegración de las economías de planificación central y el surgimiento de un modelo capitalista de carácter planetario, apoyado técnicamente por la globalización de los sistemas de comunicación. Dentro de este contexto, los agricultores pierden capacidad de desarrollar sus propias opciones y son absorbidos por las innovaciones tecnológicas de las empresas transnacionales.

[5] La distribución espacial de los montos pluviométricos en el territorio provincial experimentaron variaciones importantes en las últimas tres décadas. Las lluvias en el Oriente Chaqueño se mantenían hasta la década del '50 con valores promedios de 1000 a $1200 \mathrm{~mm}$. A partir de la década del '60 comienzan dichas oscilaciones que determinaron períodos más húmedos y que dieron lugar al incremento de las precipitaciones.

[6] Unos de los aspectos que sobresale en estos patrones productivos es la "la crisis de realización del capital y la innovación tecnológica que llevaron a reducir los ciclos de recomposición y revalorización del capital, lo cual generó procesos de producción destructiva capaces de multiplicar y acelerar la extracción de recursos no renovables" (Zarrilli, Op.Cit)

[7] Brunhes, denomina a estos tipos de actividades como "hechos de economía destructiva" asociado al nomadismo primitivo o al asentamiento humano más o menos precario. Citado en BRUNIARD, Enrique D.(1978)

[8] Se entiende por problemas a los aspectos ambientales productivos, económicos y sociales, en donde se visualizan barreras para el desarrollo sustentable de una eco-región. 
Revista Geográfica Digital. IGUNNE. Facultad de Humanidades. UNNE. Año 8. № 16. Julio - Diciembre 2011.

\section{Bibliografía}

1. BIDASECA, K; GRAS, C. y MARIOTTI, D.(2000): "Las transformaciones del mercado de trabajo en el escenario rural: viejos y nuevos procesos de negociación". En: III Congreso Latinoamericano de Sociología del Trabajo. Buenos Aires. Asociación Latinoamericana de Sociología del Trabajo.

2. BONFILS, Constante G.(1970): "Los Recursos Edáficos". En: Boletín de la Sociedad Argentina de Botánica, Volumen XI, Buenos Aires.

3. BRUNHES, Jean, 1928: La Geographie Humanine, $3^{\mathrm{a}}$ ed, Ed. F.Alcan, Paris, Francia

4. BRUNIARD, Enrique D.(1978): El Gran Chaco Argentino. Ensayos de Interpretación geográfica, GEOGRÁFICA 4, Revista del Instituto de Geografía, Fac. de Humanidades, UNNE. Resistencia, Chaco.

5. CARLEVARI, Isidro J. F (1996): La Argentina: Estructura Humana y Económica. $2^{\circ}$ ed. Buenos Aires. Ed. Macchi.

6. CORBOZ, A (2000): El territorio como palimpsesto. (Mimeo)

7. GIARRACA, Norma (1996): Procesos de globalización y cambios en la agricultura argentina. En Diego E. Piñeiro (Compilador) "Globalización, integración regional y consecuencias sociales sobre al agricultura". Universidad de la República. Uruguay.

8. GLACKEN, Clarence: 2000: Huellas en la Playa de Rodas. Ed. del Serbal

9. HARVEY, David (1998): La condición de la posmodernidad. Investigación sobre los orígenes del cambio cultural.Amorrortu editores, Buenos Aires

10. MIRANDA, Guido, 1955: Tres ciclos chaqueños. $1^{0}$ Edición. Ed. Norte Argentino, Resistencia, Chaco

11. MOYNIHAN,Daniel P., «The United States in opposition», Commentary, vol. 59, n. 3, marzo 1975 and The political and commercial works of that celebrated writer D'Avenant, LI D. ed, C. Whitworth, 5 vols., Londres, 1771, vol. I, p. 354-355. En http://www.eurosur.org/medio_ambiente/:

12. PERTILE, Viviana C. (2003): La situación social y económica del pequeño productor algodonero en el Chaco a fines de los '90, su correlato con la ampliación de la frontera agropecuaria chaqueña. Tesis de Maestría. Inédito.

13. PERTILE, Viviana C. (2004): Ampliación de la Frontera Agropecuaria Chaqueña: El Oeste chaqueño y el cultivo algodonero. En: GEOGRÁFICA DIGITAL Instituto de Geografía (IGUNNE) Facultad de Humanidades. UNNE http://hum.unne.edu.ar/revistas/geoweb/homeig0.htm

14. REBORATTI, Carlos (1999) Ambiente y sociedad. Conceptos y relaciones. Buenos Aires, Editorial Ariel, 1999. segundo semestre de 2000..Pagina Web

15. SAUER, Carl (1941): Introducción a la Geografía Histórica. Facultad de Humanidades, UNNE, Resistencia. Trad. Marta B. de Rezanowicz.

16. ZARILLI, Adrián, (2000) "Transformación ecológica y precariedad económica en una economía marginal. El Gran Chaco Argentino 1890-1950" En Theomai. Estudios sobre sociedad, naturaleza y desarrollo. CHAQUEÑO. UNA APROXIMACIÓN A LA RELACIÓN HOMBRE-NATURALEZA. Revista Geográfica Digital. IGUNNE. Facultad de Humanidades. UNNE. Año 8. № 16. Julio - Diciembre 2011. ISSN 1668-5180 Resistencia, Chaco. En: http://hum.unne.edu.ar/revistas/geoweb/default.htm 